\title{
Automated Geophysical Observatory (AGO) Lidar - Post Antarctic Deployment Performance
}

\author{
Jonathan A. R. Rall \\ John Cavanaugh ${ }^{\dagger}$ \\ James Campbell ${ }^{\dagger \dagger \dagger}$ \\ James B. Abshire \\ James D. Spinhirne ${ }^{\dagger \dagger}$ \\ Laser Remote Sensing Branch / Mail Code 924 \\ 'Lasers \& Electro-Optics Branch/Mail Code 554 \\ †Mesoscale Atmospheric Processes Branch / Mail Code 912 \\ NASA Goddard Space Flight Center \\ Greenbelt, MD 20771 \\ ${ }^{\dagger}$ Science Systems Applications Inc. \\ Phone: (301)614-6736 / Fax: (301)614-6744 / email: jrall@pop900.gsfc.nasa.gov
}

\begin{abstract}
A ground based, autonomous, low-power atmospheric lidar instrument developed at NASA Goddard Space Flight Center was recently returned from an extended period on the Antarctic polar plateau. We report on the pre and post deployment performance of AGO Lidar, compare the instrument performance with lidar models, and compare results with with the anticipated performance of the new Automatic Weather Station (AWS) Lidar.
\end{abstract}

\section{INTRODUCTION}

The compact, autonomous AGO Lidar which was deployed to the Automated Geophysical Observatory (AGO 1, E 129³6.5' Lon, S 8351.6' Lat) operated continuously for six months from January 1999 until the thermoelectric generator which powered the platform failed in early July. The instrument broadcast data from its remote location to ground stations via ARGOS/NOAA satellite network. A network of lidar instruments will be established at selected sites on the Greenland ice sheet and by co-locating them with remote Automatic Weather Station (AWS) sites in Antarctica. The NSF Office of Polar Programs provides support to place the weather stations in remote areas of Antarctica in support of meteorological research and operations. The AWS meteorological data will directly benefit the analysis of the lidar data while a network of ground based atmospheric lidar will provide knowledge regarding the temporal evolution and spatial extent of Type Ia polar stratospheric clouds (PSC). These clouds play a crucial role in the austral springtime destruction of stratospheric ozone over Antarctica, i.e. the ozone hole. ${ }^{1,2}$ In addition, the lidar will monitor and record the general atmospheric conditions (transmission and backscatter) of the overlying atmosphere which will be used for ground-truth for the $532 \mathrm{~nm}$ lidar channels of the Geoscience Laser Altimeter System (GLAS) to be launched aboard ICESat in late 2002 or early 2003 .

\section{DESIGN}

AGO Lidar, shown in Fig. 1, is a bistatic depolarization lidar. $^{3} \quad$ It uses redundant, novel semiconductor-opticalamplfier-based laser transmitters operating at a low pulse energy but a high repetition rate to achieve average powers similar to giant pulse lidar instruments. ${ }^{4}$ A commercial telescope is used to collect the backscattered photons and a polarization beam splitting cube is used to split the scattered light into two fiber optic-coupled, single photon counting modules to form the two orthogonal component polarization receiver. Custom electronics, including laser diode drivers, multi-channel scalars, temperature controllers and battery charging/monitor circuits were developed at Goddard using advanced CMOS technology to keep the continuous power draw to approximately 13 Watts. The duty cycle which is adjustable, was set to $33 \%$ while installed in AGO 1 to further reduce power consumption to 8 Watts. This was necessary due to the limited power available from the thermoelectric generator (TEG) used used in the AGO platform.

\section{A. $\quad$ Laser transmitters}

The laser transmitter design uses a $0.5 \mathrm{~W} \mathrm{CW}$ flared semiconductor optical amplifier in a Littman external cavity. A small amount of light emitted from the rear aperture of the amplifier is incident on a diffraction grating. The first order diffraction is selectively fedback into the amplifier to establish the laser cavity. With both external cavity lasers operating, the lidar transmits $>1$ microJoule (uJ) per pulse at $666 \mathrm{~nm}$ using 1 microsecond (us) pulses at $4 \mathrm{kHz}$ pulse repetition frequency (PRF). A beam expander reduces the divergence of the transmitted laser pulses to approximately 100 microradians (urad).

B. Receiver

A commercial, $20 \mathrm{~cm}$ diameter, SchmidtCassegrain telescope is used to collect the backscattered light. The telescope has been modified with a fixed focus 


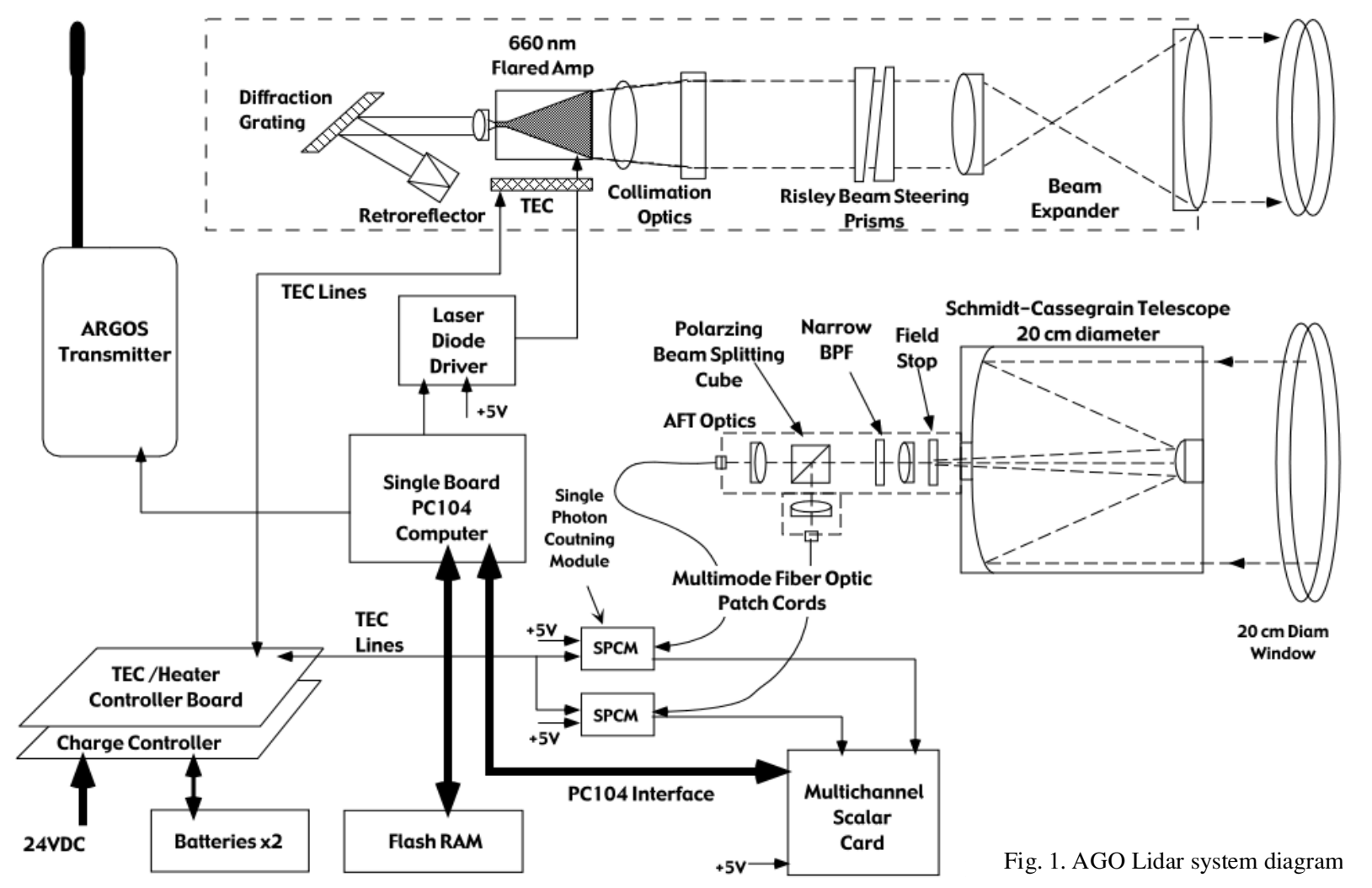

and the field of view of the receiver is limited to $140 \mathrm{urad}$ which reduces background noise and susceptibility to multiple scattering. The aft optics include a recollimation lens and a refractory-oxide, interference filter with a fullwidth, half-maximum (FWHM) bandwidth of $0.3 \mathrm{~nm}$ to further reduce background. The restricted FOV and narrow optical band pass filter permit limited daytime operation. The light is then split into orthogonal polarization components and coupled into multimode fiber optic cables which direct the light into independent photon counting modules. The output pulses produced by the detectors are collected and stored on independent multichannel scalars and later read out by a computer for analysis.

\section{RESULTS}

Typical AGO Lidar backscatter data are shown in Fig 2. Only one laser was operating, transmitting approximately $0.5 \mathrm{uJ} /$ pulse into the atmosphere and the data was acquired in 10 minute integrations with zero dead time. The lidar was operated from just before sunset January $16^{\text {th }}$ to several hour after sunrise on January $17^{\text {th }}, 2002$. After subtracting the background and range-correcting the raw data, the $\log$ of the corrected data is scaled to a 256 gray scale and plotted. The transmitted laser light is highly linearly polarized ( $>300: 1$ polarization ratio) and a polarizing beam splitting cube separates the parallel and perpendicular components and sends them to independent photon counting modules. The top plot in Fig 2 shows the parallel polarized backscattered light while the bottom plot shows the perpendicular or cross-polarized backscattered light. Fig. 3 shows the calculated depolarization ratio,

$$
\delta=\frac{I_{\perp}}{I_{\|}},
$$

where $I_{\perp}$ and $I_{\|}$are the measured perpendicular and parallel backscatter intensities. From the bottom plot in Fig. 2, the boundary layer is more strongly evident in the crosspolarized channel. This is likely due to the significant depolarization of the incident laser light caused by nonspherical aerosols which tend to accumulate on top of the boundary layer. Cloud returns are evident above $7 \mathrm{~km}$ altitude in both channels in the first four hours of the data set. However the returns are significantly stronger in the cross polarized channel which is likely due to the high ice content of the cirrus clouds or possibly jet contrails. The strong cloud returns in the troposphere from 4 to 13 hours show strong depolarization of the incident light. Given their altitude of $3-5 \mathrm{~km}$, these clouds are mostly comprised of water droplets and therefore it is likely that the strong depolarization is due to multiple scattering effects. ${ }^{5}$ After sunrise two distinct cloud layers occur and are evident in the parallel channel. However, these clouds are lost in the higher background of the cross polarized channel. This may be due to the low sun angle, the polarizing effect of the water droplets in the clouds, and the unfortunate orientation 
of the instrument. As evidenced by the top plot in Fig. 2, the parallel channel provides useful ceiliometer data during the day when background levels are higher in both channels. Further improvements to receiver spectral filtering could be done with a temperature tuned etalon. however, this would likely necessitate active control of the laser wavelength.
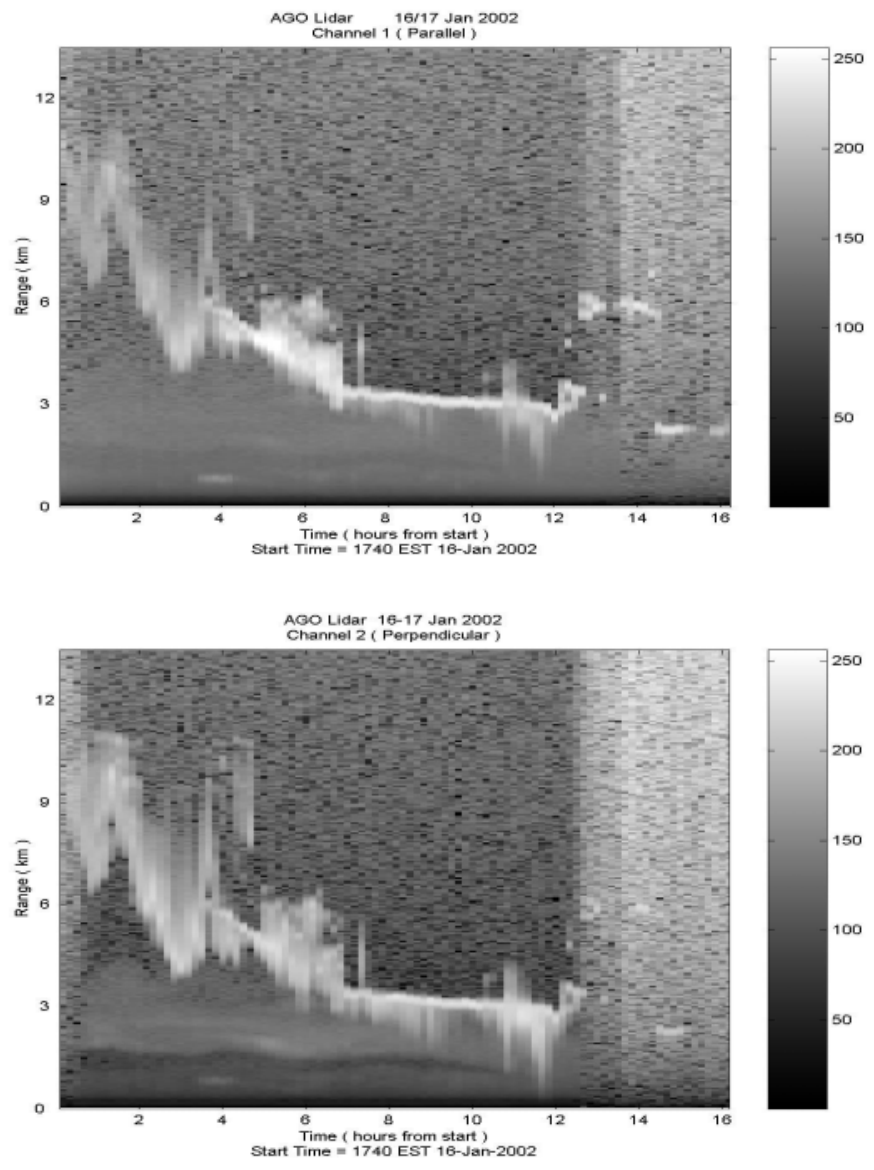

Fig. 2. Atmospheric returns from just before sunset on Jan $16^{\text {th }}$ to several hours after sunrise, Jan $17^{\text {th }}$, 2002. Top plot shows parallel polarized returns while bottom show cross polarized returns. Data was acquired in 10 minute slices (integrations).

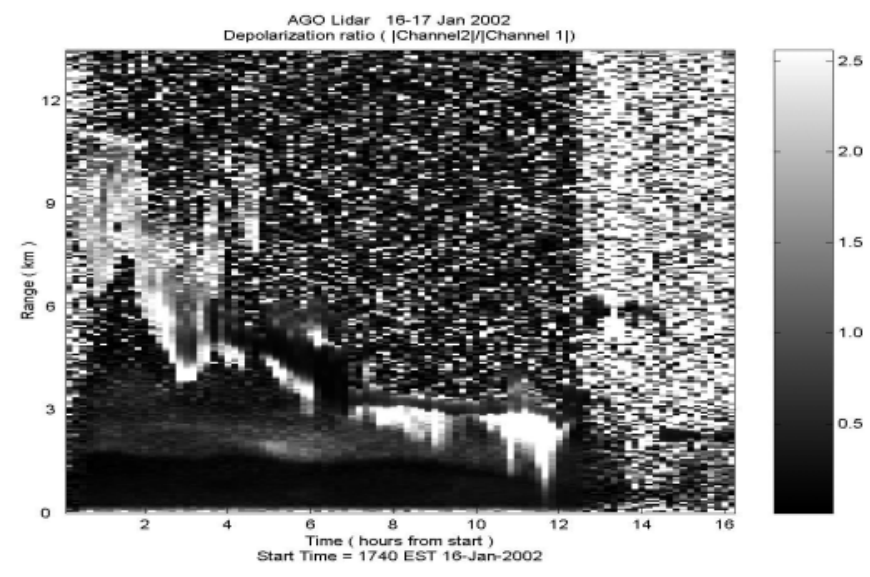

Fig. 3. Calculated depolarization from Fig. 1 shows enhanced scattering at the boundary layer.

\section{CONCLUSIONS}

The AGO Lidar has proven itself to be a rugged and robust instrument capable of performing depolarization lidar measurements of the atmosphere. It can distinguish depolarization from aerosols just above the boundary layer to depolarization from ice crystals in high altitude cirrus clouds all while consuming less than 15 watts of power. This instrument survived two years on the polar plateau, experiencing cold soaks to below $-65^{\circ} \mathrm{C}$ and the harshness of air and ground transport inherent in the U.S. Antarctic Program without significant failure. Experience gained from the AGO Lidar project has provided a solid foundation for the approach and design of the future AWS Lidar instrument.

\section{REFERENCES}

[1] M. P. McCormick, H. M. Steele, Patrick Hamill, W. P. Chu, T. J. Swissler, "Polar Stratospheric Cloud Sightings," Journal of the Atmospheric Science vol. 39, No. 6, pp. 1387-1397, 1982.

[2] Solomon, et. al., Nature vol. 321, 1986.

[3] J. A. R. Rall and James B. Abshire, "AGO Lidar / Antarctic Miniature Lidar," International Geoscience and Remote Sensing Symposium, Seattle, WA, July 6-10, 1998.

[4] J. D. Spinhirne, J. A. R. Rall, and V. S. Scott, "Compact Eye Safe Lidar System," Japanese Journal, 1995 Review of Laser Engineering.

[5] Kenneth Sassen \& Kuo-Nan Liou, "Scattering of Polarized Laser Light by Water Droplet, Mixed-Phase and Ice Crystal Clouds. Part II: Angular Depolarizing and Multiple-Scattering Behavior," Journal of the Atmospheric Sciences, vol. 36, No. 5, pp. 852-861, 1979. 